\title{
Entre historias y culebras: más que una bahía (Bahía Culebra, Guanacaste, Costa Rica)
}

\author{
Celeste Sánchez-Noguera ${ }^{1,2}$ \\ 1. Centro de Investigación en Ciencias del Mar y Limnología (CIMAR), Universidad de Costa Rica, 11501-2060 San \\ Pedro, San José, Costa Rica. \\ 2. Maestría en Gestión Integrada de Áreas Costeras Tropicales (GIACT), Universidad de Costa Rica; \\ celeste08@gmail.com.
}

Recibido 20-VI-2011. Corregido 02-XI-2011. Aceptado 10-II-2012.

\begin{abstract}
Among stories and snakes: more than a bay (Bahía Culebra, Guanacaste, Costa Rica). Bahía Culebra (Culebra Bay) is a semi-closed and protected geomorphological unit, which has a wide diversity of natural resources, encouraging the settlement of human populations and consequent coastal development. Bahía Culebra has been an important place from the historical, political, cultural and economic perspective, according to the literature search which included documents dating from the mid nineteenth century until 2011, from the National Archive of Costa Rica, the National Library, the Historical Research Center in Central America and some private collections. Its beauty, road networks, and the proximity to an international airport has made easier for it to become an intensive tourism development center. Coastal development was accelerated by the creation of the Polo Turístico Golfo de Papagayo (Papagayo Gulf Tourist Destination project). It should strengthen the legal framework and implementation of actions to promote the welfare of local populations and the proper management of natural resources, taking into account all factors and actors involved. Rev. Biol. Trop. 60 (Suppl. 2): 1-17. Epub 2012 April 01.
\end{abstract}

Key words: Culebra Bay, history, Costa Rica, coastal development.

Bahía Culebra, localizada en el Golfo de Papagayo (Fig. 1, 2), se identifica como una de las bahías más profundas y protegidas en la costa Pacífica de América Central (Lange et al. 1980, Jiménez 2001, Rodríguez-Sáenz \& Rodríguez-Fonseca 2004). Estas características geográficas aunadas a la amplia diversidad de recursos naturales, han favorecido el asentamiento de poblaciones humanas dentro de ella desde tiempos precolombinos (Lange \& AbelVidor 1980). El complejo sistema que integra la bahía en términos biológicos, sociales y económicos, la han perfilado como sitio de estudio para diversas ramas académicas. Consecuentemente existe una vasta cantidad de trabajos e investigaciones científicas desarrolladas en la zona (Cortés 2012), sin embargo destaca un vacío respecto al análisis integral de su desarrollo costero, fundamental para mejorar la gestión integrada y sostenible de sus recursos.

Para lograr una mejor comprensión de la situación actual que enfrenta la bahía, es necesario hurgar en el pasado y conocer cuál ha sido el proceso de su desarrollo. En este trabajo se recopilan fotografías y documentos históricos (documentos del Archivo Nacional de Costa Rica, investigaciones arqueológicas, publicaciones históricas y mapas, entre otros) de Bahía Culebra, y se analizan dentro del contexto de desarrollo costero experimentado en la zona, para emprender con el lector un viaje histórico a través de esta intrigante bahía. Con base en la ocupación humana documentada y las distintas fuentes de registros históricos, se definieron dos períodos de ocupación histórica y cinco de ocupación económica (Cuadro 1), delimitados 


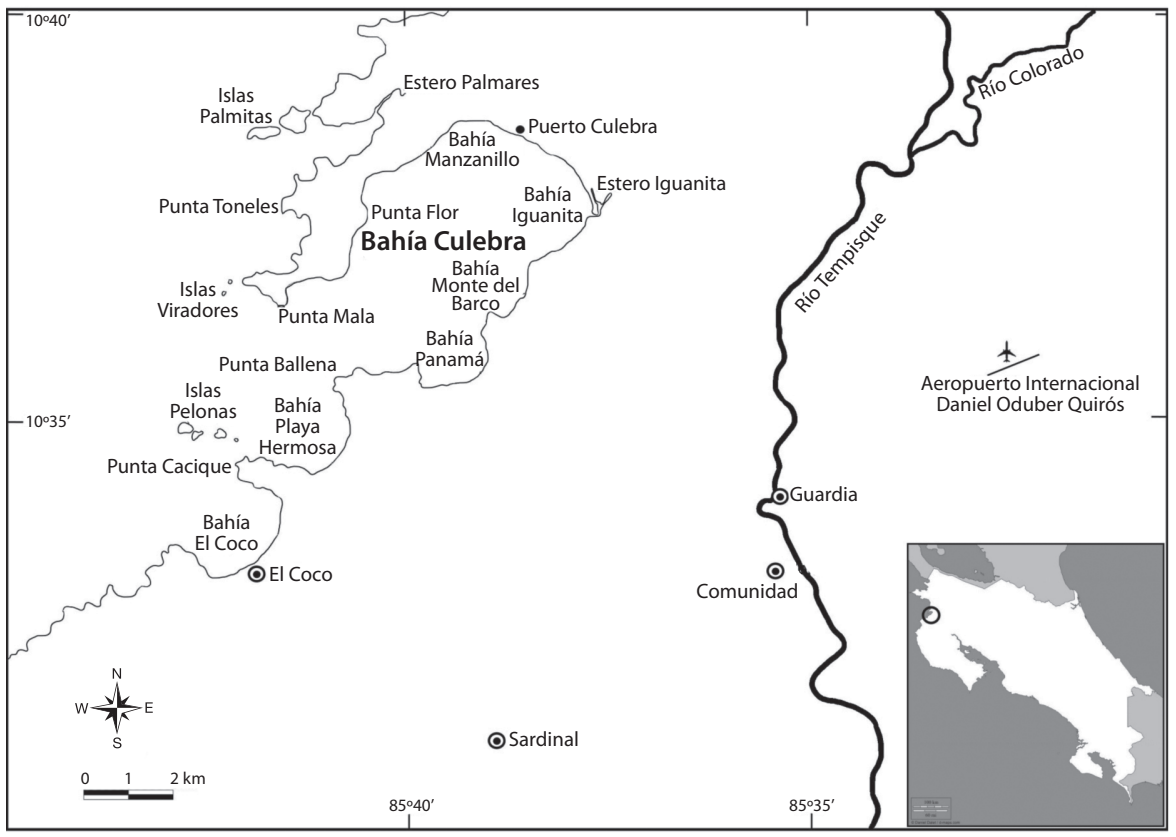

Fig. 1. Bahía Culebra, incluye pueblos limítrofes de la zona de influencia del proyecto Polo Turístico Golfo Papagayo y localización del aeropuerto internacional Daniel Oduber Quirós.

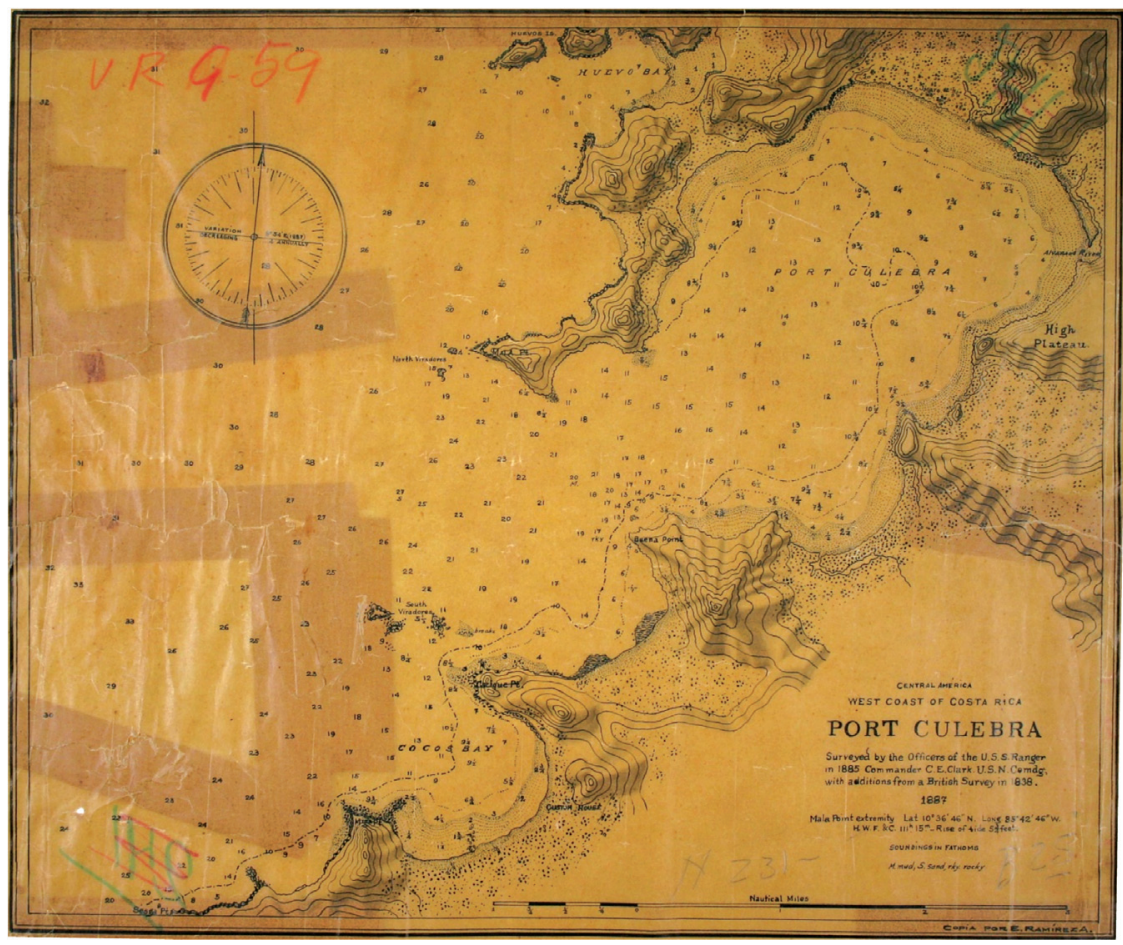

Fig. 2. Bahía Culebra a finales del siglo XIX, elaborado con datos de estudios hechos por oficiales del U.S.S Ranger en 1885 y por investigaciones inglesas en 1838 (ANCR 1887). 


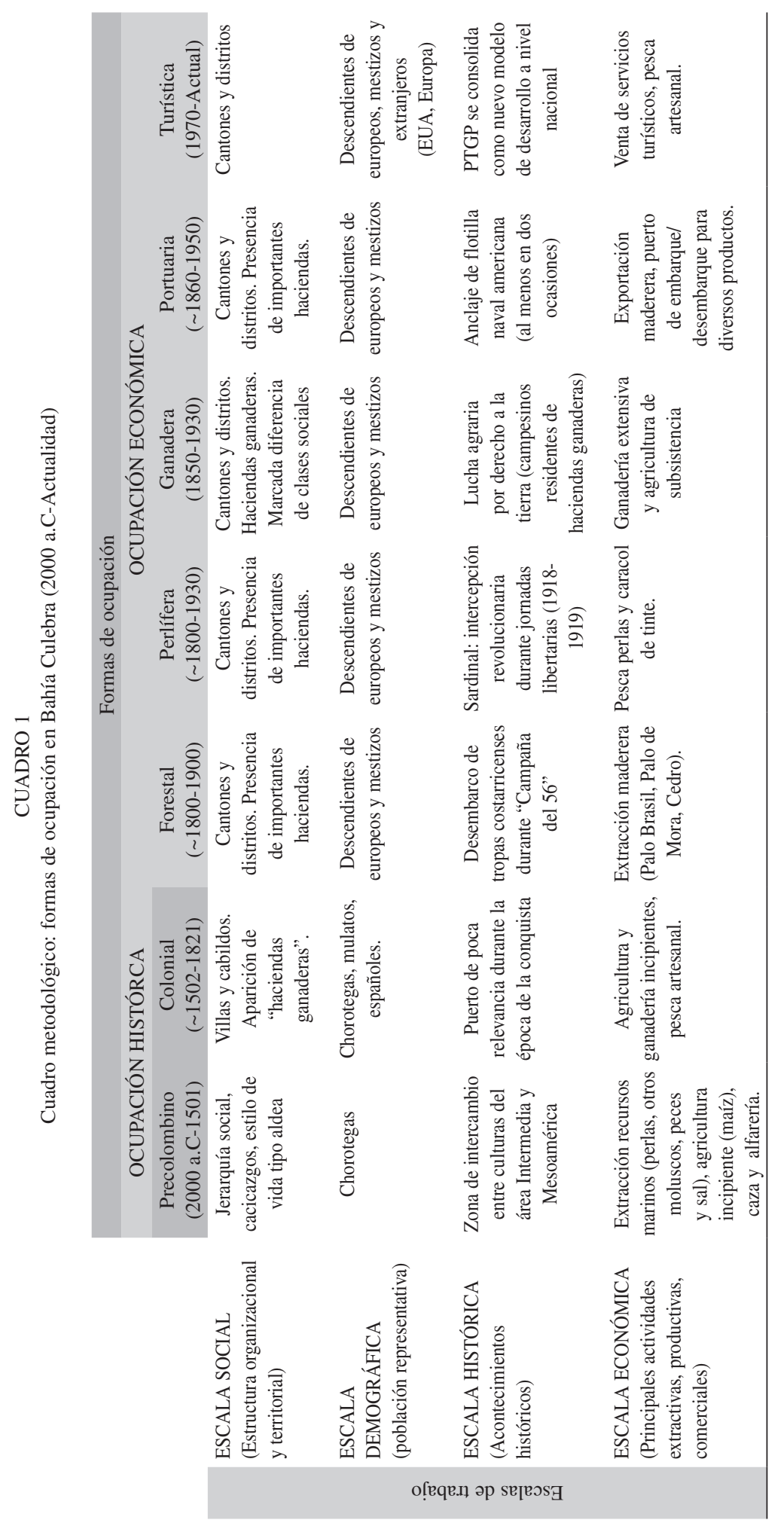


por el aprovechamiento del espacio físico de la bahía y sus recursos naturales.

Es importante tomar en cuenta que este documento constituye una breve reseña histórica del desarrollo costero que ha experimentado la bahía, así como el aprovechamiento de los recursos naturales albergados en ella. El hilo de la narración parte de algunos hallazgos que revelan situaciones propias de la época precolombina, y continúa una secuencia temporal que comprende desde el período de la colonia (siglo XVI) hasta el día de hoy (2011). Esta secuencia temporal no es estrictamente lineal, pues el esquema narrativo se desarrolla con base en los distintos usos que han experimentado la bahía y los recursos allí contenidos, además algunos de los periodos de ocupación que se definieron exhiben un traslape temporal (Cuadro 1).

\section{HISTORIA DEL DESARROLLO COSTERO}

\section{Período precolombino}

El hallazgo de evidencia arqueológica en numerosos sitios dentro de la bahía revela una ocupación intensa y permanente a partir del 800 d.C (Abel-Vidor 1980a, Lange et al. 1980), sin embargo, la recopilación de otras cronologías arqueológicas del Pacífico norte señala que posiblemente esta ocupación ha sido más extensa en la escala temporal, abarcando el periodo Orosí (2000-500 a.C) (Geocad Estudios Ambientales 2009). Los trabajos de excavación y prospección efectuados en Bahía Culebra por diferentes arqueólogos entre 1960-1980 (Lange 1980, Lange et al. 1980), permiten conjeturar que el sitio funcionó como zona de intercambio entre las culturas del área Intermedia y Mesoamérica, operando como puerto de escala o enclave comercial. Su relevancia como sitio portuario durante la época precolombina responde a dos características fundamentales, la amplitud espacial de la bahía reconocida a nivel centroamericano y el alto grado de protección contra los fuertes vientos, ambas condiciones estimularon el desarrollo de la actividad comercial.
En 1881 el funcionario de la Institución Smithsonian en Washington, D.C., John Francis Bransford, realiza el primer viaje arqueológico con fines científicos en la región, permitiéndole asociar los descubrimientos de Guanacaste con la cultura Chorotega (Meléndez 1974, NuñezArias \& Marín-Hernández 2009). Más que hallar restos arqueológicos, Bransford tuvo la valiosa oportunidad de observar las actividades que realizaban algunas indígenas, permitiéndole descifrar rápidamente la escena. Observó mujeres indígenas de Chiriquí, compañeras de los pescadores de perlas, procesando los productos extraídos por los hombres. Los desechos generados en dicha actividad posteriormente llegarían a constituir conchales, todos a pequeña escala, pero sin duda similares a los grandes conchales hallados en otros sitios arqueológicos de la bahía.

Pese a que las visitas de arqueólogos al sitio se registran desde la llegada de Bransford, la investigación intensiva inicia hasta 1973 (Lange \& Abel-Vidor 1980, Lange et al. 1980) con importantes hallazgos que revelan gran cantidad de información concerniente a las sociedades precolombinas que habitaban la zona. Por ejemplo, el patrón de sepulturas categorizadas por sexos y edades (cementerios distintos) denota una costumbre social de entierro, además algunos mostraban elaborados patrones mortuorios y contenían ofrendas suntuosas (Abel-Vidor 1980a, Wallace \& Accola 1980). El uso diferencial de los espacios funerarios en combinación con el empleo del oro y el jade en los entierros, permite deducir la existencia de un sistema de jerarquización social organizado en cacicazgos, con un estilo de vida tipo aldea (Vazquez-Leiva \& Weaver 1980, Herrera 1997). También se indica la existencia de un sitio (Monte del Barco) que probablemente fungía como campamento de abastecimiento para aldeas de mayor importancia (Accola \& Ryder 1980).

Se cree que la actividad volcánica en las cercanías de la bahía promovió los patrones de asentamiento costero observados, ya que el incremento del aprovechamiento de los recursos marinos compensaba las pérdidas en 
producción agrícola provocadas por el vulcanismo (Kerbis 1980). Así mismo, se señala que la presencia de estuarios en la zona (Iguanita y Palmares) pudo haber sido un factor fundamental en sus sistemas de subsistencia (Wallace \& Accola 1980).

A partir del estudio de restos arqueológicos y las patologías observadas en la bahía, se documentan cambios en los patrones de subsistencia, entre los distintos periodos arqueológicos reconocidos. De esta forma podía haber periodos con predominancia de la actividad agrícola, la extracción de recursos marinos o la cacería (Accola \& Ryder 1980, Lange 1980, Vazquez-Leiva \& Weaver 1980, Gómez 1997, Hernández 1997). El descubrimiento de trampas marinas especializadas para la pesca, sitios para la extracción de sal y concheros (Lange et al. 1980, Gómez 1997, Solís 1997), constituyen rastros adicionales del aprovechamiento de los recursos marinos. Además, es pertinente señalar que productos como la sal y los moluscos, también fueron importantes bienes de intercambio con otras zonas (Hernández 1997).

Es un hecho que la ardua labor arqueológica ha permitido discernir aspectos económicos y comerciales de la época (Lange 1980, Lange et al. 1980, Hernández 1997), establecer el estilo de vida precolombina predominante en la bahía (Vázquez \& Weaver 1980, Gómez 1997) y la organización social (Herrera 1997), así como los factores culturales y algunas tradiciones (Abel-Vidor 1980a, Wallace \& Accola 1980, Gómez 1997).

\section{Del período colonial a la Costa Rica contemporánea}

Luego de que el Océano Pacífico fuera reclamado en nombre del Rey y la Reina de España, el licenciado Gaspar de Espinosa es enviado en 1519 por el gobernador Pedrarias Dávila en un viaje exploratorio a través de la costa Pacífica de Panamá y Costa Rica. Hasta la fecha, el libro "Historia General y Natural de las Indias" (Fernández de Oviedo y Valdés 1853) parece ser el documento histórico más antiguo en el cual se menciona la existencia de Bahía Culebra, en él se narra que cuando Espinosa navegó hacia el Norte llegó a un lugar muy particular llamado Golfo de las Culebras: “... porque hay inumerables, que se andan sobreaguadas en la mar, de tres palmos é poco mas luengas, todas negras en los lomos, y en lo de abaxo de las barrigas todo amarillo, é de lo negro baxan unas puntas, é de lo amarillo suben otras que se abraçan unas con otras, como quien entretexiesse los dedos de las manos unos con otros, assi estas dos colores se juntan: las mas gruessas dellas son mas gordas quel dedo pulgar del pié ó como dedos de la mano juntos, é de ahí mas delgadas otras." (Fernández de Oviedo y Valdés 1853).

Otro documento histórico (ANCR 18601900) también revela que la bahía fue utilizada como puerto de entrada durante la primera etapa del período de conquista, pues señala que algunos colonizadores llegaron a sus inmediaciones luego de la visita del licenciado Espinosa. No obstante, es importante tomar en consideración las anotaciones de algunos académicos (Gerhard 1960 en Lange 1980), indicando que pese a que la bahía se encontraba localizada en las cercanías del camino colonial principal (Camino Real), su carácter portuario no fue explotado al máximo por españoles ni piratas durante la época de la conquista. Existe una ausencia significativa de información referente a la bahía durante el periodo colonial, la cual puede estar basada en la influencia marina de de los vientos "papagayos" (limitaron la entrada de los exploradores) y la escasez de población indígena organizada en pequeñas comunidades (lo cual implicaba menos recurso humano para la explotación). Hubo un lapso de tiempo entre la época precolombina y el periodo colonial, durante el cual el potencial portuario de Bahía Culebra no fue debidamente reconocido, siendo hasta finales del periodo colonial que el mismo es apreciado por los visitantes europeos (Abel-Vidor 1980b).

\section{Capital natural de la bahía}

Siglos después de la llegada de los primeros exploradores a Bahía Culebra (Fernández 
de Oviedo y Valdés 1853), el naturalista William Beebe protagoniza una visita exploratoria como parte de su viaje por distintas bahías (Beebe 1942), en la cual efectúa importantes descripciones sobre los impresionantes organismos marinos (ostrácodos, moluscos, valvas vacías del género Pinna, cangrejos, anguilas y culebras marinas) (Cortés 2012) y terrestres (el ave Caracara sp., monos carablanca (Cebus capucinus), monos aulladores (Alouatta palliata) y un felino de gran tamaño) que habitan la bahía. La abundancia y diversidad de flora y fauna constituye un importante capital natural de la zona, cuya pérdida parcial es recordada hoy en día con gran nostalgia por algunos lugareños de edad avanzada (Gutiérrez-Oviedo 2008). Los textos de Beebe también permiten confirmar la presencia de ambientes coralinos, pues entre las anécdotas mencionan haber realizado algunas labores sobre "cabezas de coral" a una profundidad promedio de un metro; asimismo las serpientes marinas de la especie Pelamis platurus obtuvieron mucha atención por parte de la tripulación, ya que nunca habían visto tantos individuos reunidos en un mismo lugar (Beebe 1942). La existencia de estos organismos en la bahía había sido señalada hacía más de cuatro siglos por Espinosa, durante sus viajes por la costa Pacífica (Fernández de Oviedo y Valdés 1853). El gran valor descriptivo de la narración detallada en sus documentos, posteriormente fue utilizada por el herpetólogo E.H. Taylor (1953) como referencia, con el fin de señalar que esta especie ha estado presente en las costas costarricenses (y por ende latinoamericanas) desde tiempos remotos.

\section{Aprovechamiento directo de los recursos}

Se documenta que en 1807, un navío Peruano arriba en la bahía con la justificación de montar una campaña extractiva del Palo Brasil (Haematoxylon brasiletto, árbol maderable de la zona). No obstante su objetivo específico iba más allá, y pretendía buscar elementos básicos para desarrollar una explotación más intensa y permanente de las riquezas agropecuarias de la región (Meléndez
1974). En este viaje se documentan una serie de observaciones, las cuales incluyen aspectos sociales y económicos, así como breves descripciones climáticas; además llevaron a cabo una ardua labor de levantamiento cartográfico de las costas.

En documentos del Archivo Nacional y algunas bitácoras de viajes se hace mención al aprovechamiento de varios recursos de la zona, sin embargo considero importante prestarle atención a cinco actividades extractivas en particular, tres de las cuales se realizaban en el mar y las otras dos en la tierra. La tala de árboles maderables era una de las principales actividades económicas llevadas a cabo en tierra firme ("Periodo Forestal", Cuadro 1), principalmente de las especies conocidas como "Palo Brasil", "Palo de Mora" (Chlorophora tinctoria) y "Cedro" (Cedrela odorata). Estos productos componían el tráfico principal en Bahía del Coco (Fradin 1892, Canales 1936a, Gutiérrez-Oviedo 2008), pero a finales del siglo XIX comenzaba a ser evidente el impacto negativo de la extracción forestal desmedida, pues las especies estaban escaseando en la bahía y era necesario viajar hasta orillas del Tempisque para la labor de corta. En esa época Puerto Culebra funcionó como uno de los principales sitios para el embarque y desembarque de maderas (ANCR 1884, 1910). Se cree que muchas de las exportaciones tenían como destino final Sur América (Canales 1936a, Gutiérrez-Oviedo 2008), hecho que podría confirmarse parcialmente si detallamos que la Fragata Joaquina (Meléndez 1974) venía en representación del Comercio del Perú. Posteriormente, se halla evidencia de la explotación de recursos minerales, mediante denuncias que se presentaron por la existencia de minas de oro, plata y cobre abandonadas (ANCR 1899), todas ubicadas en zonas aledañas a la bahía.

Con respecto a los recursos marinos, destaca la extracción del caracol de tinte Plicopurpura patula pansa (utilizado para la tinción de tejidos) y la pesca de perlas (Pinctada mazatlanica), esta última considerada “...la más famosa de todas las costas del Océano Pacífico" (Canales 1936a, Meléndez 1974) ("Periodo 
Perlífero, Cuadro 1). En otros registros (Fradin 1892, Zamora 1924) continúa presente esta actividad extractiva, pues se habla de sitios ricos en concha perla, ubicados en Bahía del Coco y Bahía Venado. La extracción de perlas y del caracol de tinte fueron actividades que coexistieron durante varios periodos históricos (incluso mucho antes de la colonia), pues se acostumbraba realizarlas simultáneamente (Payne-Iglesias 2008). Finalmente, la pesca artesanal de fauna íctica constituye una actividad extractiva documentada desde el periodo precolombino, la cual continúa vigente hoy en día, pese a que ya no constituye el eje principal de la economía local pues ha sido desplazada por el modelo turístico (Cuadro 1).

Con este recuento de algunas de las principales actividades económicas, se demuestra que históricamente la extracción de recursos en Bahía Culebra ha tenido lugar en el mar, la costa y la tierra.

\section{Desarrollo portuario de Bahía Culebra}

En el período de 1891-1892, el capitán de la Marina Francesa Eliseo Fradin, realiza estudios sobre la factibilidad de instalar otro puerto en Bahía Culebra, ya que el puerto existente (Puerto Culebra; Marín-Hernández \& NúñezArias 2011a) presentaba problemas de acceso e inundaciones durante la época lluviosa (Fradin 1892). Luego de explorar la totalidad de la bahía y generar descripciones físico-oceanográficas sumamente valiosas, Fradin (1892) la califica como una zona óptima para la creación de un Puerto de Guerra y Comercio, argumentando que "...está en capacidad de abrigar la armada más grande de Europa". Así mismo, señala otras características favorables para que la bahía se desempeñe como puerto, entre las cuales menciona la escasez de arrecifes (probablemente haciendo referencia a las formaciones rocosas, pues los señalaba como peligrosos obstáculos para la navegación) y la abundancia de buenos fondeaderos y bahías que permiten desembarcar fácilmente. Finalmente, destaca el hecho de que la mayoría de las bahías internas están rodeadas por valles y terrenos aptos para el cultivo, aunado al fácil acceso por tierra que tienen algunos de los sitos.

Durante la exploración del Allan Hancock por el Océano Pacífico del Este (Fraser 1943) (Fig. 3), esta bahía recibe el calificativo de ser la mejor de Centroamérica para el anclaje de barcos de gran calado. Esta cualidad particular que distintos exploradores le atribuyeron a Bahía Culebra es corroborada en 1923, cuando una flotilla naval americana arribó y permaneció ahí por varios días (Zamora 1924). La

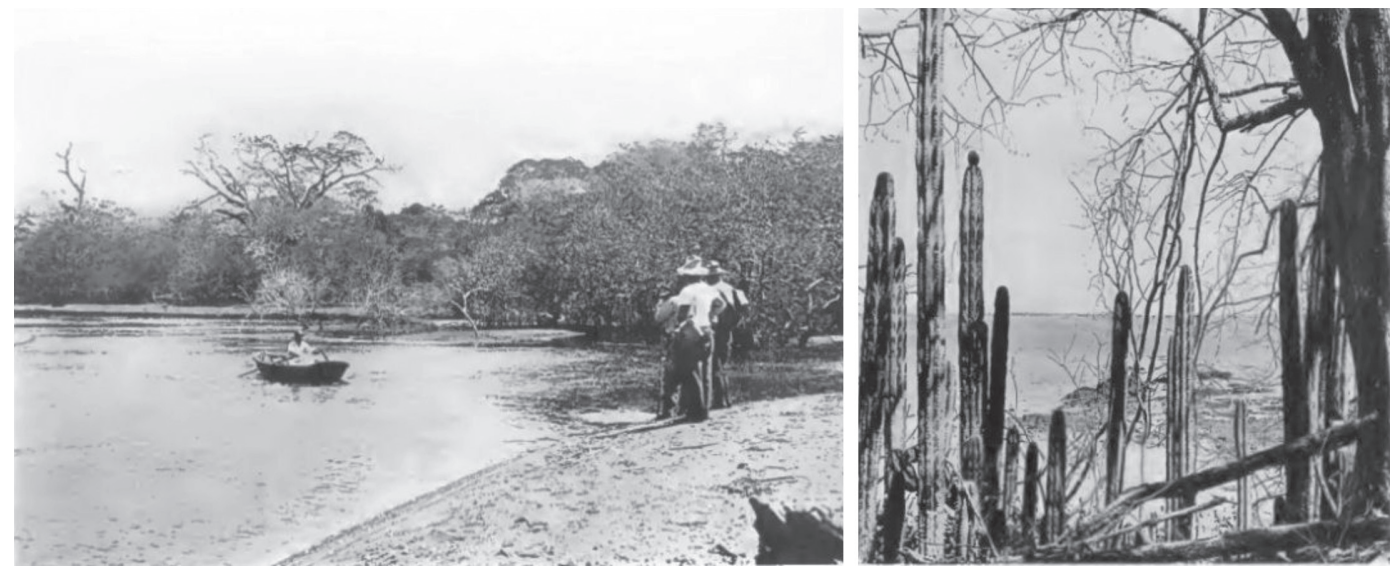

Fig. 3. Bahía Culebra, durante la visita del Velero III en su viaje por el Pacífico Oriental, 1931-1941. Izq: "Estuario en Puerto Culebra, Costa Rica, muestra una canoa utilizada para movilizarse entre los estrechos canales de agua rodeados de mangle", Der: "Puerto Culebra, Costa Rica, vista desde atrás de una densa vegetación de cactáceas columnares" (tomado de Fraser 1943, con autorización de la unidad de colecciones especiales de la Universidad del Sur de California (USC)). 
colección personal de Luis Escalante (com. pers.) permite confirmar la presencia de estos buques, pues cuenta con una serie de fotografías que muestran la flotilla anclada en la bahía (Fig. 4), así como un sello postal del portaviones USS Lexington enviado desde Bahía Culebra, con fecha 12 de mayo de 1934. Este conjunto de documentos históricos (textos, artículos de periódicos, fotografías y sellos postales) permiten confirmar la presencia de barcos de la Marina de los Estados Unidos dentro de la bahía en al menos dos ocasiones. De la primera visita (1923) se desconocen los motivos, no obstante es un hecho documentado que los locales se sorprendieron de su presencia y de la potencia de sus reflectores que irradiaban a varios kilómetros de distancia (Zamora 1924). Con respecto a la segunda visita, se logró corroborar que correspondió a un simulacro de toma del Canal de Panamá, en el cual las escuadras estadounidenses del Pacífico y el Atlántico tuvieron como lugar de defensa las bahías de Culebra y del Coco, con presencia de 70 barcos de guerra de gran calado, hidroaviones y muchísimos marineros (Canales 1936b) (Fig. 5).

Luego de examinar las innumerables cualidades que calificaban a Bahía Culebra como un sitio óptimo para el establecimiento de un puerto, surgen varios decretos que buscan oficializar esta situación, tanto en algunas de sus bahías internas como en aquellas aledañas a Bahía Culebra con características igualmente favorables. Así, en $1878 \mathrm{El}$ Coco es declarado Puerto de Depósito y se demanda el establecimiento de una Aduana de Registro, con el objetivo que opere para depositar toda clase de mercaderías pagando un pequeño impuesto de
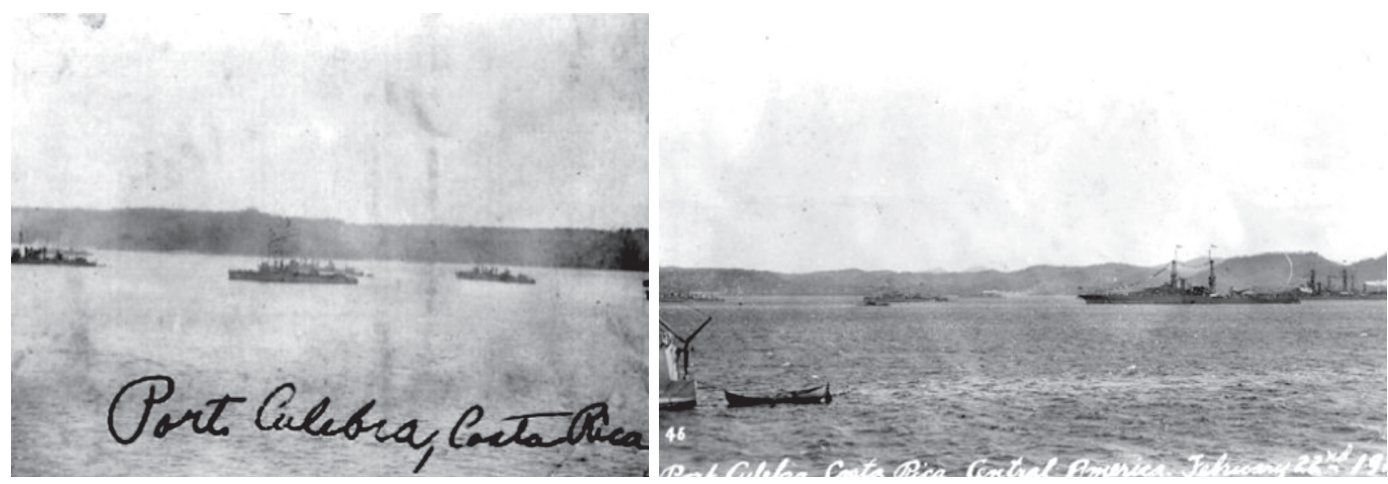

Fig. 4. Flotillas de buques navales estadounidenses ancladas en Bahía Culebra, año 1923 (originales pertenecen a la colección personal de Luis Escalante).
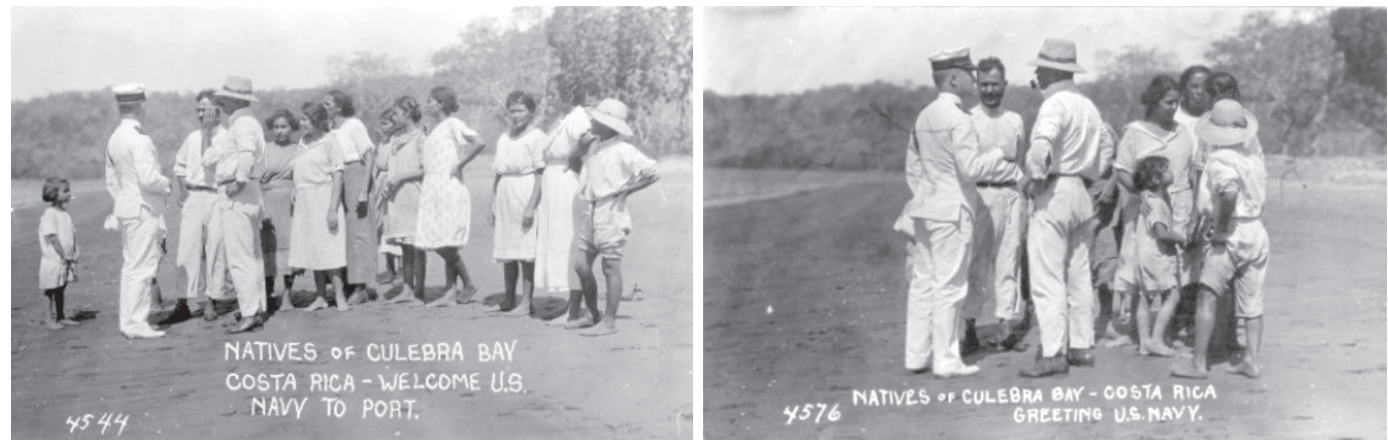

Fig. 5. Recibimiento de los miembros de la marina estadounidense por parte de los habitantes de Bahía Culebra, a inicios de la década de 1920 (originales pertenecen a la colección personal de Álvaro Castro-Harrigan). 
bodegaje (ANCR 1878). El establecimiento de dicho puerto implicaría un gran beneficio para el comercio local, no obstante es importante destacar que dicha acción estaba inmersa dentro de un panorama más amplio, la inclusión de la provincia de Guanacaste en los procesos sociales a nivel nacional (Núñez-Arias \& Marín-Hernández 2009).

Las recomendaciones específicas elaboradas por Fradin (1892), luego de analizar cada una de las playas y bahías contenidas dentro de Bahía Culebra, favorecen la decisión de declarar un puerto de altura en Bahía Panamá (ANCR 1894). No obstante, esta declaratoria es modificada años más tarde (ANCR 1915) y se resuelve que este puerto también debe establecerse en El Coco. Dentro de la historia del cabotaje guanacasteco, los puertos inicialmente se insertaron con el comercio de maderas, consolidándose posteriormente con el modelo agroexportador. Sin embargo, datos históricos de distintas fuentes (Marín-Hernández \& Núñez-Arias 2011b) señalan que El Coco se estancó en la primera etapa del sistema de cabotaje (maderera).

En un mensaje presidencial de 1913, don Ricardo Jiménez manifiesta lo favorable que resultaría activar el puerto del Coco para exportar los productos provinciales (Marín-Hernández \& Núñez-Arias 2011b). Esto evidencia que la decisión política de establecer el puerto de altura en El Coco, responde a la urgente necesidad de explotar las riquezas naturales de la zona y diversificar las actividades económicas, pues hasta la fecha estas se limitaban a la industria pecuaria y la explotación maderera. Al declarar este sitio como puerto de altura sería indispensable la habilitación de nuevas vías de comunicación, situación que favorecería el crecimiento empresarial de la localidad y a los consumidores, pues contarían con una mayor oferta de productos a un menor costo (el transporte desde Puntarenas encarecía los productos). En síntesis, podría decirse que habilitar estos puertos mejoraría las importaciones y exportaciones tanto en las inmediaciones de la bahía como en zonas vecinas, y pese a estas visibles ventajas económicas resulta paradójico que transcurrieron casi 20 años (ANCR 1915, Canales 1936a, b, c) antes de que se decretara su establecimiento y la construcción de la carretera que los comunicaría con la ciudad de Liberia.

\section{Eventos históricos de relevancia nacional}

Bahía Culebra y sus zonas aledañas también desempeñaron un papel fundamental en los acontecimientos históricos del país, destacando que durante la "Campaña del 56" (contienda nacional para evitar la invasión por parte del filibustero William Walker en 1856; Calvo 2006) cuando las tropas costarricenses se dirigían a Nicaragua para enfrentar al ejército de William Walker, se vieron forzados a desembarcar en Bahía Culebra y continuar su travesía por tierra (ANCR 1856a, b). El poblado de Sardinal es memorable en la historia del país, debido a la contienda que ahí se libró durante esta Campaña. Además fue un punto estratégico para interceptar revolucionarios y prevenir la invasión durante las jornadas libertarias de 1918-1919 (Zamora 1924).

\section{La "hacienda ganadera”}

A nivel provincial la ganadería comenzó a consolidarse como la actividad económica principal desde el siglo XVIII, y es a partir de este momento que surge la figura conocida como "hacienda ganadera". Esta nueva forma de producción se caracterizaba por la delimitación de grandes territorios dedicados a la ganadería extensiva, en manos de un único propietario (o de una familia). En sus inicios se adquirían esclavos para trabajar en las haciendas, y posteriormente estos trabajadores se constituyeron en una clase asalariada de escasos recursos. A mediados del siglo XIX las haciendas ganaderas conformaron un importante sistema de tenencia y explotación agrícola, presentes en casi todo Guanacaste y en las zonas aledañas a la bahía. De este modo, se sabe que existían al menos dos importantes "haciendas ganaderas" en las inmediaciones de Bahía Culebra: Hacienda Culebra y Hacienda Las Trancas (Sequeira-Ruiz 1985). 
La aparición de la "hacienda ganadera" implicó el desmantelamiento de la propiedad agraria indígena existente, así como el sistema de organización social y cultural asociado a ella (Gutiérrez-Oviedo 2008). A nivel socioeconómico, este periodo se caracterizó por grandes brechas en el ámbito social, con unos pocos con mucha riqueza y poder, y una mayoría desposeída. El grado de pobreza era distinto al identificado actualmente, pues aunque no se veían precarios, sí había una notoria diferencia en la calidad de la alimentación de los hacendados con respecto a los empleados. El fortalecimiento de la "hacienda ganadera" también implicó una pérdida del capital forestal de la bahía y la modificación del paisaje natural, pues vino acompañado de un incremento en la deforestación del bosque seco para expandir las haciendas y abrir nuevos potreros (Vargas 2011).

Durante el periodo de 1930-1970 ocurrió una transición del modelo productivo de la "hacienda ganadera" a la agroindustria, motivo por el cual se definió que la ocupación económica ganadera de la bahía contemplaría únicamente el periodo comprendido entre 1850 y 1930 (Cuadro 1). Con el modelo de producción agroindustrial surge una modernización y tecnificación del agro, caracterizada por la inclusión de nueva maquinaria en las labores agrícolas, por ejemplo los tractores. De igual forma se diversifican los cultivos, dando paso a la siembra de nuevos productos como la caña, el algodón, el arroz y el sorgo. Esto conllevó a la reducción en las áreas dedicadas al pasto para abrir paso al desarrollo de los nuevos cultivos, no obstante, este cambio no implicó una disminución del hato, sino que promovió un mayor aprovechamiento de los terrenos dedicados a la actividad ganadera (GutiérrezOviedo 2008).

\section{Desarrollo turístico: \\ el nuevo eje de la economía local}

Dejando a un lado el estudio específico de las maravillas naturales contenidas en la bahía, cabe mencionar que su riqueza paisajística (Fig. 6) también ha sido un recurso altamente explotado, especialmente en las últimas décadas. La construcción de la carretera interamericana fue un factor muy importante en la historia de la región Guanacasteca, ya que implicó un cambio significativo en la red vial y por lo tanto en el acceso a la zona, dando como resultado final un incremento en el turismo (Zeledón-Torres 2009). Según compilaciones de la prensa escrita, desde la década de 1930 se están redoblando esfuerzos para promocionar la provincia como destino turístico, exaltando la belleza de sus paisajes, su riqueza cultural y amplia gama de opciones turísticas para todos los presupuestos (Editorial 1935, Zúñiga 1936). Esos escritos hacen particular enfoque en Playas del Coco (Acuña de Chacón 1935, El

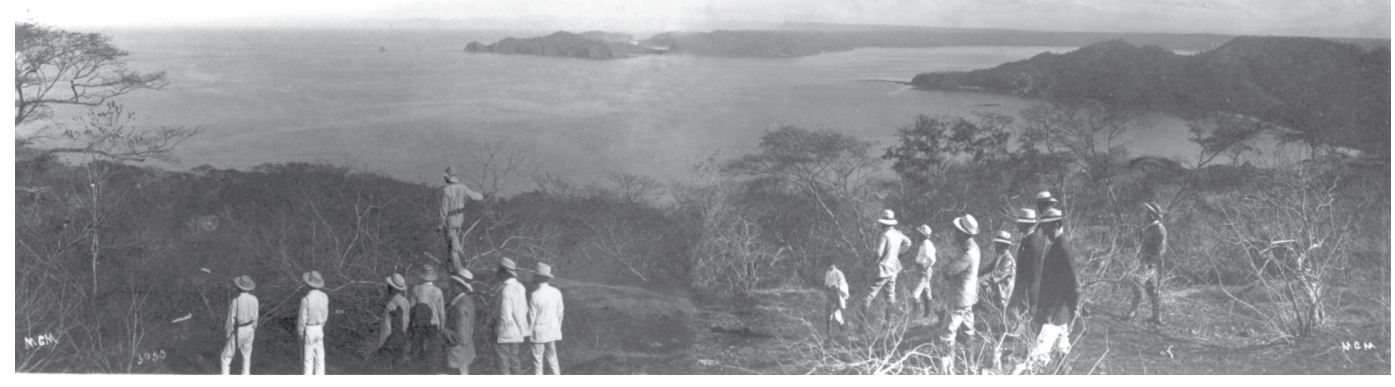

Fig. 6. Vista de Bahía Culebra y Naranjo desde el Cerro Cacique. Fotografía tomada por Manuel Gómez Miralles durante la gira presidencial de Alfredo González Flores a Guanacaste en 1916 (CEDOCIHAC 2011). 
Guanacaste 1936) y la playa en Bahía Manzanillo (Fig. 1). Esta última fue la primera opción, luego de un estudio regional, para construir el primer hotel de una cadena extranjera (ArrietaMurillo \& Rivera-Hernández 2009).

En términos turísticos, Bahía Culebra se convirtió en un modelo de desarrollo distinto, bajo el esquema planteado por el proyecto Polo Turístico Golfo de Papagayo (PTGP), cuyo diseño original data de la década de 1970, pero se comienza a implementar hasta 1991 con el otorgamiento de la primera concesión (Salas 2010, V. Cajiao 2012). Dicho proyecto nace en respuesta a las políticas nacionales que buscaban desarrollar el turismo internacional, y se perfila como un modelo nuevo en Costa Rica, cuyo manejo es público-estatal con miras a la aprobación de concesiones de capital extranjero (ICT 1980, Lange et al. 1980, Alonso 2009). El creciente interés del Estado por impulsar dicho proyecto, motiva que el mismo sea declarado de utilidad pública en 1979 (Poder Legislativo 1979). Después de transcurridos más de 30 años de su concepción se declara de conveniencia nacional (Poder Ejecutivo 2006).

En la década de 1980 el proyecto PTGP es eje central en las discusiones sobre el futuro del turismo en el país, especialmente por los atrasos en su implementación. Según Arrieta-Murillo \& Rivera-Hernández (2009) "Guanacaste como provincia, se convierte en sustantivo condicionado de Papagayo, pues no es la región como un todo lo que importa, sino el apéndice representado por el enclave". Esto deja al descubierto que en realidad el proyecto fue visto desde sus inicios como la solución inmediata para los problemas socio-económicos que enfrentaba la provincia. Dados los inconvenientes que surgieron con la implementación del proyecto, surge la necesidad de crear una Ley que regule su desarrollo y ejecución, la cual es publicada en 1982 (Poder Legislativo 1982). Cinco años después de entrada en vigencia esta Ley, el mandatario a cargo, Dr. Oscar Arias Sánchez, le otorga al sector privado poder de mando administrativo en la zona, de manera no oficial, al manifestar que considera que este sector será el llamado a realizar y administrar el proyecto (ANCR 1987). A partir de ese momento se ceden espacios para que la administración de la bahía esté en manos del sector privado, hecho que a largo plazo podría acarrear repercusiones negativas en el ámbito social, cultural y ambiental.

\section{PANORAMA ACTUAL (1990-2011)}

Luego de este breve recorrido histórico de Bahía Culebra, llegamos a los hechos actuales que allí acontecen. Ciertamente el proyecto PTGP ha generado amplias expectativas en los habitantes de la zona, pero a su vez ha estado acompañado de polémicas e inconvenientes desde el momento en que fue concebido. En 1991 se planteó que los megaproyectos resultan desfavorables para la provincia guanacasteca, por lo cual se analizó el impacto del proyecto Polo Turístico Golfo de Papagayo (ArrietaMurillo \& Rivera-Hernández 2009).

La influencia que ha ejercido este proyecto sobre el área se evidencia en cambios muy tangibles, como son la cantidad de infraestructura turística (hoteles, marinas, locales comerciales y enfocados en la venta de servicios turísticos) y habitacional (condominios) construida en los últimos años, el desarrollo de actividades orientadas exclusivamente al turismo (pesca deportiva, buceo recreativo, observación de cetáceos, alquiler de motos acuáticas, entre otras) y el crecimiento económico que ha experimentado el poblado de Playas del Coco, localizado dentro del área de influencia del proyecto (ICT 1980, Cascante-Gutiérrez et al. 1994).

La extensión del proyecto PTGP en línea costera ronda los $26.33 \mathrm{~km}$, con un área aproximada de 15.120.951,96 $\mathrm{m}^{2}$ (poco más de 1,500 ha) (Oficina Ejecutora PTGP). Desde que se inició la ejecución del proyecto es evidente que ha habido un cambio en el uso urbano, con incrementos en el área destinada para la construcción de facilidades turísticas y habitacionales, las cuales no pueden exceder el $30 \%$ de su densidad según lo establecido en el Plan Maestro (ICT 1995). No obstante, en la actualidad existe un vacío de información respecto al desarrollo inmobiliario, y en la oficina 
ejecutora del proyecto en el Instituto Costarricense de Turismo (ICT), no cuentan con datos actualizados sobre el área de construcción y los porcentajes de expansión (H. Wong com. pers. 2011). Claramente esto puede vislumbrarse como un problema durante el análisis del proyecto, pues implica una falta de control para corroborar el cumplimiento de los lineamientos estipulados en el Plan Maestro.

Lo que sí existe son registros actualizados de las concesiones ubicadas en el área del proyecto. Según archivos del ICT, a marzo 2011, dentro de la bahía existe un total de 28 concesiones, con registro de 10 hoteles y operadores comerciales (Hotel Four Seasons, Exclusive Resort, Marina Papagayo S.A., Hotel Alegro Resort Papagayo, Hotel Hilton Papagayo, Hotel Giardini Di Papagayo, Hotel Resort Conde del Mar, Sol Papagayo Resort, Condo Hotel Monarca y Occidental Resort Grand Papagayo). Se considera un logro relevante para el desarrollo del PTGP la inauguración de la Marina Papagayo en diciembre del 2008, ya que dicho proyecto estaba comprendido dentro del Plan Maestro de la concesionaria Ecodesarrollo Papagayo (Camacho-Sandoval 2010).

La ejecución del proyecto PTGP se ha enfrentado a un sinfín de dificultades desde su creación, no sólo por ser un novedoso modelo de desarrollo en el país que planteaba nuevos retos, sino también porque en algunos casos se han tomado decisiones político-administrativas que han desencadenado conflictos entre los diferentes usuarios y actores. Tal es el caso del Decreto Ejecutivo No 35962 ("Reforma Reglamento a la Ley para el Desarrollo y Ejecución del Proyecto Turístico Golfo de Papagayo") (Poder Ejecutivo 2010) firmado por el ex mandatario Dr. Oscar Arias Sánchez, el cual generará pérdidas monetarias sustanciales a las municipalidades de Liberia y Carrillo. Con dicho decreto se reduce el canon que deben pagar las distintas concesionarias, al modificar el cálculo del gravamen: ya no se basará en el valor real de la propiedad ocupada por el desarrollo turístico, sino en un valor fijado por el ICT (correspondiente a $\$ 1.56 / \mathrm{m}^{2}$ indexado).
Las pérdidas económicas que generará este nuevo decreto, aunado al descontento popular por considerar que esta decisión estuvo basada en intereses personales (Anónimo 2011a, b, Mejía 2011), han motivado críticas y denuncias por parte de diputados y regidores municipales (Anónimo 2011a, b, Mejía 2011, Villegas 2011) para solicitar la derogación del mismo.

Pese a que los lineamientos establecidos en el Plan Maestro del PTGP lo perfilan como un modelo de desarrollo turístico sostenible, "al establecer un desarrollo debidamente planificado, bajo administración y supervisión estatal, el cual incorpora variables ambientales, bajas densidades y coberturas, tratamiento adecuado de aguas servidas, etc" (ICT 1995, Poder Ejecutivo 2006), a la lista de controversias y noticias nacionales se suman ciertas irregularidades de tipo ambiental denunciadas hace algunos años (Salas 2010, Arce 2011, Salazar 2011), así como otras relacionadas con el entorpecimiento de acceso a playas públicas (Sala Constitucional 2008). En un esfuerzo reciente por subsanar algunos errores cometidos, el ICT le canceló el derecho de concesión a una sociedad anónima y sancionó a otras cuatro firmas desarrolladoras, debido a incumplimientos en el contrato (Arce 2011). Aunado a esto es importante destacar que en el 2004 (Poder Ejecutivo 2004) se declaró de interés prioritario el desarrollo de proyectos de investigación, protección y educación ambiental, como una iniciativa para promover la conservación y el uso racional de los recursos marinos en el Golfo de Papagayo y sus zonas de influencia. Sin embargo, hasta la fecha sólo se tiene conocimiento de un programa de investigación financiado por una concesionaria, en este caso Ecodesarrollo S.A (J. Cortés com. pers. ). El fundamento legal por sí solo no garantiza el manejo adecuado de los recursos, resulta indispensable el compromiso por parte de todos los usuarios, así como una vigilancia más exhaustiva por parte de las entidades coresponsables de la administración de la bahía (municipalidades de Liberia y Carillo) y el proyecto (ICT). 


\section{CONCLUSIONES}

Su morfología costera permite calificar a Bahía Culebra como una bahía semicerrada, bastante amplia y protegida (Jiménez 2001, Rodríguez-Sáenz \& Rodríguez-Fonseca 2004), dentro de la cual se ha favorecido el asentamiento de grupos humanos desde tiempos precolombinos (Lange \& Abel-Vidor 1980, Lange et al. 1980). Como todo asentamiento humano esto conllevó la alteración de la costa, lo cual viene acompañado de la explotación del espacio y los recursos naturales, para satisfacer las necesidades básicas de alimento, abrigo y ocio. A medida que el desarrollo se expande, comienzan a surgir nuevos usos de los recursos en respuesta a las nuevas necesidades de la sociedad, sin embargo, la historia muestra una extracción intensiva y un uso desmedido que en conjunto han promovido su agotamiento (e.g. perlas, palo Brasil, palo de Mora y Cedro) y deterioro (e.g. ambientes marinos en general).

Con esta recapitulación histórica se espera que quede claro que la importancia de la bahía ha trascendido en varios ámbitos, algunos hechos que ejemplifican esto, ocurridos entre el período precolombino y finales del siglo XX son: la pesca y extracción de sal llevada a cabo durante la época precolombina, su función como puerto de entrada para exploradores y colonos durante el período colonial (Fernández de Oviedo y Valdés 1853, ANCR 1860-1900), destaca la extracción de recursos marinos y terrestres posterior al periodo colonial (Fradin 1892, ANCR 1894, 1899, 1910, Canales 1936b, Beebe 1942, Meléndez 1974, Gutiérrez-Oviedo 2008), a nivel nacional fungió como puerto significativo en las importaciones y exportaciones (ANCR 1878, 1884, 1910, Fradin 1892, Canales 1936b), además fue un punto estratégico en la Campaña de 1856 (ANCR 1856a, b) y en otros períodos de la historia para interceptar revolucionarios (ANCR 1854, Zamora 1924). Finalmente, se comprobó que la protección que brinda Bahía Culebra resultó estratégica para el anclaje de grandes barcos (Canales 1936b) y flotillas navales (Zamora 1924, Canales 1936c, L. Escalante com. pers.).
Desde finales del siglo XIX se ha vislumbrado a Bahía Culebra como un sitio de grandes desarrollos, primero se pensó únicamente en términos de desarrollo portuario, pero con el pasar de los años la idea evolucionó hacia un concepto más completo que incluía el desarrollo turístico. La diversidad de recursos marinos que alberga la bahía se convirtió en un importante capital económico para distintos sectores, principalmente el sector pesquero artesanal y el turístico. Su notoria belleza escénica, la disponibilidad de redes viales en buen estado y la cercanía al aeropuerto internacional Daniel Oduber Quirós (ubicado en Liberia, Fig. 1), facilitaron que la bahía se convirtiera en un centro turístico intensivo en los últimos años, generando un crecimiento acelerado en su desarrollo costero. Esto surge a raíz de la creación del proyecto Polo Turístico Golfo de Papagayo en la década de 1970, cuyo manejo pretendía impulsar políticas nacionales para desarrollar la región como un polo de turismo internacional (Salas 2010) y se convirtió en un nuevo modelo de desarrollo. En el sector turístico la explotación de los recursos se ha manifestado tanto de forma directa como indirecta, lo cual ha desencadenado una serie de conflictos de intereses entre sus principales usuarios (Ibarra-Gené 1996, Jiménez 1997, Dominici-Arosemena et al. 2005).

Es evidente que a lo largo de la historia se han tomado algunas decisiones políticas claves para el desarrollo de la región, como fue la habilitación de los puertos que mejoraría el comercio en toda la provincia. No obstante, debe reconocerse que otras decisiones han servido como catalizador para conflictos entre los usuarios, a la vez que potencian el riesgo de un crecimiento descontrolado, tal es el caso del Decreto Ejecutivo No. 35962 ("Reforma Reglamento a la Ley para el Desarrollo y Ejecución del Proyecto Turístico Golfo de Papagayo"). Es importante continuar con la labor de fortalecimiento del marco jurídico, pero sin perder de vista que el norte de estos esfuerzos deberá dirigirse a mejorar la calidad de vida de las comunidades locales como también cimentar las bases para una adecuada gestión de los 
recursos naturales, tomando en cuenta todos los factores y actores involucrados. Todavía queda mucho trabajo por hacer, para lograr reconciliar la utilización de los recursos con los intereses de los distintos sectores, procurando minimizar el impacto negativo que esto pueda generar, tanto en términos ambientales, socioeconómicos y culturales.

Esta reconciliación debe ser una labor conjunta, así como la administración del desarrollo costero en toda Bahía Culebra, bautizada desde inicios del siglo pasado como la "joya del Pacífico" (Canales 1936b). No me queda duda al respecto sobre esta necesidad, particularmente cuando regresa a mi memoria el recuerdo sensitivo de aquél primer verano en la bahía, que me hizo enamorarme de tan maravilloso lugar:

$$
\begin{gathered}
\text { “...cuando el viento juguetea } \\
\text { sigilosamente con las olas, } \\
\text { y aflora un espectro de nuevas } \\
\text { sensaciones en el mar: } \\
\text { fríos penetrantes que ascienden } \\
\text { de las profundidades, } \\
\text { dejando a su paso una estela de } \\
\text { diminutos organismos... } \\
\text {...nueva vida...” }
\end{gathered}
$$

\section{AGRADECIMIENTOS}

Mis más sinceros agradecimientos a todas aquellas personas que colaboraron de una $u$ otra manera durante la ardua labor de recopilación bibliográfica, y que de igual manera hicieron aportes significativos para la investigación. Gracias a Luko Hilje, Luis Escalante, Álvaro Castro-Harrigan, Roberto Kopper, Carlos Jiménez, Elizet Payne, Ronny Viales y Archivo Nacional de Costa Rica.

\section{RESUMEN}

Bahía Culebra (Golfo de Papagayo, Guanacaste, Costa Rica) es una unidad geomorfológica semicerrada y bastante protegida, que cuenta con una amplia diversidad de recursos naturales, favoreciendo el asentamiento de poblaciones humanas y su consecuente desarrollo marinocostero. La reseña histórica de Bahía Culebra indica que este ha sido un sitio muy importante desde la perspectiva histórica, política, cultural y económica. La consulta bibliográfica incluyó documentos que datan de mediados del siglo XIX hasta el año 2011, los cuales fueron recopilados del Archivo Nacional de Costa Rica, la Biblioteca Nacional, el Centro de Documentación del Centro de Investigaciones Históricas de América Central y algunas colecciones privadas. Su notoria belleza escénica, la disponibilidad de redes viales en buen estado y la cercanía a un aeropuerto internacional han facilitado que la bahía se convierta en un centro turístico intensivo, generando un acelerado desarrollo costero a raíz de la creación del proyecto Polo Turístico Golfo de Papagayo (PTGP). Se debe fortalecer el marco jurídico e implementar acciones que promuevan el bienestar de las poblaciones locales y la adecuada gestión de los recursos naturales, tomando en cuenta todos los factores y actores involucrados.

Palabras clave: Bahía Culebra, historia, Costa Rica, desarrollo costero

\section{REFERENCIAS}

Abel-Vidor, S. 1980a. Dos hornos precolombinos en el sitio Vidor Bahía Culebra, Guanacaste. Vínculos 6: 43-50.

Abel-Vidor, S. 1980b. The historical sources for the greater Nicoya archaeological sub-area. Vínculos 6: 155-169.

Accola, R. \& P. Ryder. 1980. Excavaciones en el sitio Monte del Barco, Bahía Culebra. Vínculos 6: 67-79.

Acuña de Chacón, A. 1935. De la vida guanacasteca: En la Playa del Coco. Periódico El Guanacaste, Guanacaste, Costa Rica, Año I (15): 2 (1ºtiembre 1935).

Alonso, S. 2009. Sección III: Desarrollo local Península Papagayo, p. 195-212. In L. Sariego, S. Alonso, M. Dierckxsens, M. Gonzalo, K. López, J. Sariego, J. Valenciano \& M. Van Lidth (eds.). Alianzas para el desarrollo: motor de la responsabilidad Social-Casos de organizaciones públicas y privadas en Costa Rica. ALIARSE, San José, Costa Rica. (también disponible en línea: http://www.aliarse.org/documentos/ALIARSE\%20completo\%20-\%20version\%20digital.pdf)

ANCR. 1854. Gobernación, No. 26067. Archivo Nacional de Costa Rica, San José, Costa Rica.

ANCR. 1856a. Secretaría de Guerra y Marina, No. 8807. Archivo Nacional de Costa Rica, San José, Costa Rica.

ANCR. 1856b. Secretaría de Guerra y Marina, No. 8911. Archivo Nacional de Costa Rica, San José, Costa Rica. 
ANCR. 1860-1900. Álbum de Figueroa (colección de documentos históricos), Tomo I, folio 65v. Archivo Nacional de Costa Rica, San José, Costa Rica.

ANCR. 1878. Archivos del Congreso, No. 8499. Archivo Nacional de Costa Rica, San José, Costa Rica.

ANCR. 1884. Ministerio de Hacienda, No. 30063. Archivo Nacional de Costa Rica, San José, Costa Rica.

ANCR. 1887. Mapas, No.7226. Archivo Nacional de Costa Rica, San José, Costa Rica.

ANCR. 1894. Archivos del Congreso, No. 2370. Archivo Nacional de Costa Rica, San José, Costa Rica.

ANCR. 1899. Juzgado Contencioso Administrativo, No. 6973. Archivo Nacional de Costa Rica, San José, Costa Rica.

ANCR. 1910. Secretaría de Policía, No. 4195. Archivo Nacional de Costa Rica, San José, Costa Rica.

ANCR. 1915. Archivos del Congreso, No. 11005. Archivo Nacional de Costa Rica, San José, Costa Rica.

ANCR. 1987. Presidencia, No. 3498. Archivo Nacional de Costa Rica, San José, Costa Rica.

Arce, S. 2011. ICT cancela una concesión de Papagayo y sanciona a cuatro. La Nación, San José, Costa Rica, 10 de enero del 2011, pág. 28 (también disponible en línea: http://www.nacion.com/2011-01-10/Economia/ NotasSecundarias/Economia2642199.aspx)

Arrieta-Murillo, G. \& G. Rivera-Hernández. 2009. El desarrollo del turismo en Guanacaste: de la Asociación Bella Vista al Instituto Costarricense de Turismo, p. 137-156. In: R. Nuñez-Arias \& J. Marín-Hernández (eds.). Historia de la (Re)Construcción de una región. 1850-2007. Alma Máter, San José, Costa Rica.

Beebe, W. 1942. Book of Bays. Barcourt, Brace \& Company, Nueva York, Nueva York.

Cajiao, M.V. 2012. Aspectos legales del Polo Turístico Golfo de Papagayo, Guanacaste, Costa Rica: régimen especial. Rev. Biol. Trop. 60 (Supl. 2): 225-230.

Calvo, J. 2006. La Campaña Nacional contra los filibusteros en 1856 y 1857: breve reseña histórica. Editorial UCR, San José, Costa Rica.

Camacho-Sandoval, C. 2010. Papagayo confiesa sus pecados. Periódico El Financiero, No 775: 4-6 (5-11 julio 2010).

Canales, M. 1936a. La Bahía del Coco habilitada como puerto de altura en las costas del Pacífico, será el vigoroso resurgimiento del Guanacaste. Periódico El Guanacaste, Guanacaste, Costa Rica, Año II (48): 7 (23 julio 1936).

Canales, M. 1936b. La Bahía del Coco habilitada como puerto de altura en las costas del Pacífico será el vigoroso resurgimiento del Guanacaste. Periódico El Guanacaste, Guanacaste, Costa Rica, Año II (49): 1 y 5 ( $1^{\circ}$ agosto 1936).

Canales, M. 1936c. La bahía El Coco habilitada como puerto de altura en las costas Norte del Pacífico, será para Guanacaste su total resurgimiento. Periódico El Guanacaste, Guanacaste, Costa Rica, Año II (47): 6 (15 julio 1936).

Cascante-Gutiérrez, A., N. Cerna-Gutiérrez, J. ChavarríaLópez, D. Díaz-Chavarría \& C. Gutiérrez-Álvarez. 1994. El turismo y su contribución al mejoramiento de las condiciones socioeconómicas de las comunidades: casos Playas del Coco, Carrillo, Guanacaste. Tesis de Licenciatura en Trabajo Social, Universidad de Costa Rica, San José, Costa Rica.

CEDOCIHAC. 2011. Colección Alfredo González Flores: Gira presidencial a Guanacaste, 1916. Centro de Documentación del Centro de Investigaciones Históricas de América Central, San José, Costa Rica.

Cortés, J. 2012. Historia de la investigación marina en Bahía Culebra, Guanacaste, Costa Rica. Rev. Biol. Trop. 60 (Supl. 2): 19-37.

Dominici-Arosemena, A., E. Brugnoli-Olivera, J. CortésNúñez, H. Molina-Ureña \& M. Quesada-Alpizar. 2005. Community structure of Eastern Pacific reef fishes (Gulf of Papagayo, Costa Rica). Tecnociencia 7 (2): 19-41.

Editorial. 1935. El Paraíso de Mahoma. Periódico El Guanacaste, Guanacaste, Costa Rica, Año I (3): 1 (1 ${ }^{\circ}$ marzo 1935).

El Guanacaste. 1936. Anuncio turístico de Playa del Coco. Periódico El Guanacaste, Guanacaste, Costa Rica, Año II (67): 1 y 5 (15 diciembre 1936).

Fernández de Oviedo y Valdés, G. 1853. Historia General y Natural de las Indias, Islas y Tierra-Firme del Mar Océano. (Publicado por José Amador de los Ríos). Imprenta de la Real Academia de la Historia. Madrid. Tomo III, lib. XXIX, cap. XIII.

Fradin, E. 1892. Estudios del Golfo de Nicoya, de la Bahía de Cocos y del Golfo de Culebra. Tipografía Nacional, San José, Costa Rica.

Fraser, C.M. 1943. General account of the scientific work of the Velero III in the eastern Pacific, 1931-1941, 
Part II: Geographic and biological associations. Allan Hancock Pac. Exped. 1: 49-258.

Geocad Estudios Ambientales. 2009. Informe Final Estudio Integral sobre el impacto de las construcciones y el desarrollo turístico y urbanístico en la Zona de Amortiguamiento del Parque Nacional Marino Las Baulas. Capítulo VII: Arqueología. GEOCAD, San José, Costa Rica.

Gómez, I. 1997. El Conchal: reflejo de la vida cotidiana. Vínculos 22: 45-78.

Gutiérrez-Oviedo, H. 2008. Me lo dijo el río: relatos breves sobre la historia de las comunidades vecinas al Polo Turístico Golfo de Papagayo 1930-1970. Edit. Universidad de Costa Rica, San José, Costa Rica.

Hernández, M.A. 1997. Manzanillo: sitio costero multicomponente en la Bahía Culebra, Guanacaste. Vínculos 22: 79-123.

Herrera, A. 1997. Espacio y objetos funerarios en la distinción de rango social en finca Linares. Vínculos 22: $125-156$.

Ibarra-Gené, E. 1996. El valor de uso del paisaje submarino en el Golfo de Papagayo: comparación de la industria de buceo recreativo con la industria de extracción de peces para acuario. Tesis de Licenciatura en Economía, Universidad de Costa Rica, San José, Costa Rica.

ICT. 1980. Proyecto Golfo Papagayo: información general. Instituto Costarricense de Turismo (ICT), San José, Costa Rica.

ICT. 1995. Reglamento No 4572: Reglamento "Plan Maestro General del Proyecto Turístico Golfo de Papagayo". Publicado en La Gaceta $\mathrm{N}^{\circ} 140$ el 24 de julio de 1995: 35

Jiménez, C.E. 1997. Corals and coral reefs of Culebra Bay, Pacific coast of Costa Rica: Anarchy in the reef. Proc. 8th Int. Coral Reef Symp., Panamá 1: 329-334.

Jiménez, C. 2001. Arrecifes y ambientes coralinos de Bahía Culebra, Pacífico de Costa Rica: aspectos biológicos, económico-recreativos y de manejo. Rev. Biol. Trop. 49 (Supl. 2): 215-231.

Kerbis, J. 1980. The analysis of faunal remains from the Vidor site. Vínculos 6: 125-140.

Lange, F. 1980. Una ocupación del policromo tardío en sitio Ruíz, cerca de Bahía Culebra. Vínculos 6: 81-96.
Lange, F. \& S. Abel-Vidor. 1980. Investigaciones arqueológicas en la zona de Bahía Culebra, Costa Rica (19731979). Vínculos 6: 5-8.

Lange, F., R. Accola \& P. Ryder. 1980. La administración de los recursos culturales en Bahía Culebra: Un informe sobre la prospección realizada dentro de la zona de impacto del Desarrollo Turístico Bahía Culebra. Vínculos 6: 9-32.

Marín-Hernández, J. \& R. Núñez-Arias. 2011a. El cabotaje guanacasteco, un análisis de sociabilidad: 1950-1960, p. 151-178. In: R. Núñez-Arias \& J. Marín-Hernández (eds.). (Re)Lecturas de Guanacaste: 1821-2010. Alquimia 2000, San José, Costa Rica.

Marín-Hernández, J. \& R. Núñez-Arias. 2011b. Los sistemas de cabotaje de Guanacaste, en un análisis comparado de articulaciones nodales interregionales en Costa Rica: 1890-2000, p. 71-122. In: R. NúñezArias \& J. Marín-Hernández (eds.). (Re)Lecturas de Guanacaste: 1821-2010. Alquimia 2000, San José, Costa Rica.

Meléndez, C. 1974. Viajeros por Guanacaste. Ministerio de Cultura, Juventud y Deportes. San José, Costa Rica.

Núñez-Arias, R. \& J. Marín-Hernández. 2009. Acotando espacios: Control estatal en Guanacaste 1860-1940, construyendo el poder en Guanacaste, p. 1-52. In: R. Núñez-Arias \& J. Marín-Hernández (eds.). Historia de la (Re)Construcción de una región. 1850-2007. Alma Máter, San José, Costa Rica.

Payne-Iglesias, E. 2008. Las perlas de la reina: explotación perlífera en el Pacífico de Centroamérica (15221850). Diálogos (Revista electrónica de Historia), Número Especial: 631-654.

Poder Ejecutivo. 2004. Decreto Ejecutivo No 32051: Declara de interés prioritario el desarrollo de proyectos de investigación, protección y educación ambiental, en procura de la conservación y uso racional de los recursos marinos existentes en el Golfo de Papagayo y sus zonas de influencia. La Gaceta No 206 el 21 de octubre del 2004: 2.

Poder Ejecutivo. 2006. Decreto Ejecutivo No 33132: "Declara de conveniencia nacional, el desarrollo y ejecución del Proyecto Turístico Golfo de Papagayo". La Gaceta No 97 el 22 de Mayo del 2006: 9.

Poder Ejecutivo. 2010. Decreto Ejecutivo No 35962 : Reforma Reglamento a la Ley para el Desarrollo y Ejecución del Proyecto Turístico Golfo de Papagayo. La Gaceta No 103 el 28 de Mayo del 2010: 4-7.

Poder Legislativo. 1979. Ley $N^{\circ}$ 6370: Declara de Interés Público el Proyecto de Bahía Culebra o Papagayo. 
Colección de leyes y decretos Semestre II (Tomo 2): 786 .

Poder Legislativo. 1982. Ley $N^{\circ}$ 6758: Regula la Ejecución del Proyecto Turístico de Papagayo. La Gaceta $N^{\circ}$ 122 el 25 de Junio de 1982: 1.

Rodríguez-Sáenz, K. \& J. Rodríguez-Fonseca. 2004. Avistamientos del delfín manchado, Stenella attenuata (Cetacea: Delphinidae) en Bahía Culebra, Costa Rica, 1999-2000. Rev. Biol. Trop. 52 (Supl. 2): 189-193.

Salas, A. 2010. Polo turístico Golfo de Papagayo, Guanacaste, Costa Rica: Análisis del Polo Turístico Golfo de Papagayo como un modelo único gubernamental de concesión turística. Center for Responsible Travel, Stanford University. San José, Costa Rica (también disponible en línea: http://www.responsibletravel.org/ resources/documents/Coastal-tourism-documents/ Polo_Tur\%C3\%ADstico_Golfo_de_Papagayo_Guanacaste_Costa\%20Rica.pdf).

Sequeira-Ruiz, W. 1985. La hacienda ganadera en Guanacaste: aspectos económicos y sociales 1850-1900. Edit. UNED, San José, Costa Rica.

Solís, F. 1997. Nuevos datos en la arqueología de Bahía Culebra, Noroeste de Costa Rica. Vínculos 22: 1-44.

Taylor, E.H. 1953. Early records of the seasnake Pelamis platurus in Latin America. Copeia 1953: 124.

Vargas, G. 2011. Del paisaje natural a los paisajes humanizados en Guanacaste S.XVII, XVIII, XIX, p. 29-52. In: R. Núñez-Arias \& J. Marín-Hernández (eds.). (Re)Lecturas de Guanacaste: 1821-2010. Alquimia 2000, San José, Costa Rica.

Vázquez-Leiva, R. \& D. Weaver. 1980. Un análisis osteológico para el reconocimiento de las condiciones de vida en sitio Vidor. Vínculos 6: 97-106.

Villegas, J. 2011. Diputado pide anular decreto que baja canon para Papagayo. La Nación, San José, Costa Rica, 19 de febrero del 2011, pág. 10 (también disponible en línea: http://www.nacion.com/2011-02-19/ ElPais/NotasSecundarias/ElPais2688903.aspx).
Wallace, H. \& R. Accola. 1980. Investigaciones arqueológicas preliminares de Nacascolo, Bahía Culebra, Costa Rica. Vínculos 6: 51-65.

Zamora, H. 1924. Sardinal, Golfo de Culebra y Bahía del Coco. Revista de Costa Rica, Año V: 200-201.

Zeledón-Torres, F. 2009. La construcción de la carretera Inter-Americana en Guanacaste estado, región y medios de comunicación 1943 y 1970, p. 101-136. In: R. Núñez-Arias \& J. Marín-Hernández (eds.). Historia de la (Re)Construcción de una región. 1850-2007. Alma Máter, San José, Costa Rica.

Zúñiga, D. 1936. Conoce Ud. Guanacaste...? Periódico El Guanacaste, Guanacaste, Costa Rica, Año II (27):1 y 4 ( $1^{\circ}$ enero 1936).

\section{Referencias de Internet}

Anónimo. 2011a. Decreto de Arias favorece concesiones de Papagayo, denuncia diputado Villalta. Diario El País, Costa Rica (Consultado 8 febrero 2011, http://www. elpais.cr/articulos.php?id=40170)

Anónimo. 2011b. Concejo de Liberia pide derogar decreto favorable a concesionarios de Papagayo. Diario El País, Costa Rica (Consultado 8 febrero 2011, http:// www.elpais.cr/articulos.php?id=41460)

Mejía, A. 2011. Diputado denuncia que decreto de expresidente Arias causó pérdidas millonarias en Papagayo. Diario El Pregón, Costa Rica (Consultado 8 febrero 2011, http://www.elpregon.org/ costarica/1-politica/3516-diputado-denuncia-quedecreto-de-expresidente-arias-a-favor-de-consecionarios-causo-perdidas-millonarias-en-papagallos).

Sala Constitucional. 2008. Voto 018915-2008, Recurso Hábeas Corpus (Consultado $1^{\circ}$ abril 2011, https://pjenlinea.poder-judicial.go.cr/SistemaGestionEnLinea/Publica/wfpDetResolucion. aspx $? \mathrm{id}=5351555 \& \mathrm{~d}=\& \mathrm{dr}=$ ).

Salazar, C. 2011. Papagayo Norte se desarrolla violando legislación ambiental de Costa Rica. Diario El País, Costa Rica (Consultado 8 febrero 2011, http://www. elpais.cr/articulos.php?id=41534). 
